\title{
Pedigree analysis for the rare RHD DVa allele: serological and molecular studies
}

\author{
Jingjing Gao ${ }^{1}$, Xiongpeng Zhu ${ }^{2}$, Jinzhe Tan ${ }^{3}$, Mingquan Wang ${ }^{1}$, Wenqian Xu ${ }^{1}$, Jhy sheng Chang ${ }^{4 *}$ \\ ${ }^{1}$ Department of Clinical Transfusion, Affiliated Quanzhou First Hospital of Fujian Medical University, Quanzhou 362000, Fujian, China; \\ ${ }^{2}$ Department of Haematology, Affiliated Quanzhou First Hospital of Fujian Medical University, Quanzhou 362000, Fujian, China; \\ ${ }^{3}$ Department of Clinical Transfusion, West China Hospital, West China School of Medicine, Sichuan University, Chengdu \\ 610041, Sichuan, China; \\ ${ }^{4}$ Department of Clinical Transfusion, National Taiwan University Hospital, Taipei 100002, Taiwan.
}

\begin{abstract}
Until now, worldwide more than 80 different alleles producing weak D phenotypes have been identified. Here we identified rare RHD DVa alleles in Chinese individuals associated with weak expression of D antigen and an RHD phenotype resembling DVI. Multi-monoclonal anti-D antibodies were used to identify the RHD phenotyping for rare RHD DVa. RHD genotyping was used to confirm the presence of RHD exons and identify RHD, RHCE hybrids and exon deficiencies. Sanger sequencing was used to identify nucleotide polymorphisms in RHD exons. Pedigree analysis demonstrated RHD DVa allele alterations of $667 \mathrm{~T}>\mathrm{G}, 676 \mathrm{G}>\mathrm{C}, 697 \mathrm{G}>\mathrm{C}, 712 \mathrm{G}>\mathrm{A}, 733 \mathrm{G}>\mathrm{C}, 744 \mathrm{C}>\mathrm{T}$ and $1227 \mathrm{G}>\mathrm{A}$, which means the proband's alleles were RHD DVa-3 [also called RHD-CE(5)-D] and $1227 \mathrm{G}>\mathrm{A}$. The results also demonstrated RHD DVa and the original RHD Va allele without $1227 \mathrm{G}>\mathrm{A}$. The study suggests that RHD phenotyping is a superior strategy for the molecular analysis of RHD variant in Chinese subjects, and for understanding related polymorphisms and mutations.
\end{abstract}

Keywords: RHD Va, Del, pedigree analysis

\section{INTRODUCTION}

The RH blood group is comprised of the most important and complicated human erythrocytic antigens, with over 50 related antigens, including RHD, RHC, RHc, RHE, and $\mathrm{RHe}^{[1]}$. The RHD antigen is the most clinically significant antigen in the RH blood group system in clinical transfusion and obstetrics. RHD variants display inconsistent reactions to different monoclonal anti-D antibodies, which include weak D, partial D and D elution, of which molecular analyses have identified more than 150 RHD variant types ${ }^{[2-5]}$.
In this study, we investigated the molecular genetic basis of the apparently weak D phenotypes observed in a proband and his family members, and described a rare RHD DVa allele.

\section{MATERIALS AND METHODS}

\section{Subject}

A 46 years old male presented for routine physical examination. Occult blood in his stool was found positive. His health condition was nice without diabetes mellitus, hypertension and other metabolic dis-

\footnotetext{
*Correspondence to: Jhy sheng Chang, Department of Clinical Transfusion, National Taiwan University Hospital, Taipei 100002, Taiwan. E-mail: jschang@seed.net.tw.

Conflict of interests: The authors declared no conflict of interests.
} 
eases, and he was deemed generally healthy. Due to the proband's family history of colorectal carcinoma having stool occult blood, the colonoscopy was arranged.

\section{Serological typing}

ABO RH typing was done by using the Wadiana automated system (Grifols Diagnositic. S.A., Barcelona, Spain) and the ABO-RHCDE typing card (Grifols Diagnositic. S.A.), which specifically identifies DVI. Antibody screening was done by using human anti-globin and micro-column testing combined to use Panels 1-3 (Grifols Diagnositic. S.A.). The antiD confirmation test followed the National Health Commission' specification for Clinical Transfusion Technology. Phenotyping involved the use of monoclonal IgG/IgM mixed anti-D (Anti-D series 4, Gamma-clone, Immucor Inc., USA) comprising of an IgM clone (MS201) and IgG clone (MS26); an antiD blend (Gamma-clone, Immucor Inc., USA) comprising of an IgM clone (GAMA401) and IgG clone (F8D8); and a Gamma-clone comprising of an anti-D (IgM clone: D175-2, and IgG clone: D4151E4; Nova- clone; Dominion Biologicals Ltd., Dartmouth, Nova Scotia, Canada).

\section{Genotyping and sequencing of RHD exons}

DNA was isolated from EDTA-anticoagulated blood using commercial kits (DNA Extraction Kit magnetic beads, ZongJi WanTai Bio-pharmaceutical Co. Ltd., Jiangsu, China) based on automated Chemagic ${ }^{\mathrm{TM}}$ magnetic separation (FluoVia Gene Pure Plus; ZongJi WanTai Bio-pharmaceutical Co. Ltd.). All DNA samples were stored at $-80^{\circ} \mathrm{C}$ until molecular analysis. The sequencing of exon 1-10 of the RHD gene and RHD genotype were analyzed using commercial kits (RHD fluo-genotyping and RHD exons sequence, ZongJi WanTai Bio-pharmaceutical Co. Ltd.).

\section{RESULTS}

\section{Serological typing}

The serological typing results are presented in Table 1 and Table 2. The RHD phenotype in the proband was determined to be an RHD variant.

Table 1 Serological ABO and RHD typing in the proband

\begin{tabular}{lcccccccccccc}
\hline Subject & Anti-A & Anti-B & Anti-D $\left(\mathrm{DVI}^{-}\right)$ & Anti-D $\left(\mathrm{DVI}^{+}\right)$ & A cell & B cell & RHC & RHE & Screen I & Screen II & Screen III \\
\hline Proband & 0 & $4+$ & 0 & $2+$ & $4+$ & 0 & $4+$ & $4+$ & 0 & 0 & 0 \\
Wife & 0 & 0 & $4+$ & $4+$ & $4+$ & $4+$ & $4+$ & $4+$ & 0 & 0 & 0 \\
Daughter & 0 & $4+$ & $4+$ & $4+$ & $4+$ & 0 & $4+$ & $4+$ & 0 & 0 & 0 \\
\hline
\end{tabular}

Table 2 RHD confirmation test in the proband

\begin{tabular}{lcccc}
\hline \multirow{2}{*}{$\begin{array}{c}\text { Anti-D } \\
\text { IgM clone: MS201 }\end{array}$} & $\begin{array}{c}\text { Anti-D } \\
\text { IgG clone: MS26 }\end{array}$ & $\begin{array}{c}\text { IgM clone: GAMA401 } \\
\text { IgG clone: F8D8 }\end{array}$ & $\begin{array}{c}\text { Anti-D } \\
\text { IgM clone: D175-2 } \\
\text { IgG clone: D4151E4 }\end{array}$ & $\begin{array}{c}\text { clone: Absorption } \\
\text { clone: Elution Test }\end{array}$ \\
\hline Proband & $2+$ & $2+$ & $2+$ & $2+$ \\
Wife & $4+$ & $4+$ & $4+$ & $/$ \\
Daughter & $4+$ & $4+$ & $4+$ & $/$ \\
\hline \multicolumn{2}{c}{ Note: The absorption elution test was based on acid elution and multiple polyclonal anti-D from RHD-pregnant women. The titer of anti-D was } \\
1:128.
\end{tabular}

\section{Molecular analysis of RHD gene}

The RHD exon genotype contained exons 1 to 10 , which primarily rule out the RHD and RHCE hybrid, leaving two RHD variant alleles. Molecular analysis of the proband demonstrated heterogenous single nucleotide polymorphisms consisting of $667 \mathrm{~T}>\mathrm{G}, 676$ $\mathrm{G}>\mathrm{C}, 697 \mathrm{G}>\mathrm{C}, 712 \mathrm{G}>\mathrm{A}, 733 \mathrm{G}>\mathrm{C}, 744 \mathrm{C}>\mathrm{T}$ and 1227 G>A (Fig.1). The RHD allele in the proband's daughter was heterogeneous only for $1227 \mathrm{G}>\mathrm{A}$, which also known as RHD*01EL.01. Pedigree analysis revealed $667 \mathrm{~T}>\mathrm{G}, 676 \mathrm{G}>\mathrm{C}, 697 \mathrm{G}>\mathrm{C}, 712 \mathrm{G}>\mathrm{A}$, $733 \mathrm{G}>\mathrm{C}$ and $744 \mathrm{C}>\mathrm{T}$ in the RHD allele, which was also an RHCE exon 5 sequence (Fig.2). This result also concluded the allele, as an RHD-RHCE(5)-RHD hybrid, also called DVa-3 (RHD*05.03).

\section{DISCUSSION}

The $\mathrm{D}$ antigen is the most clinically significant antigen of the $\mathrm{RH}$ blood group system in clinical transfusion and obstetrics. There are many $\mathrm{D}$ variants, including weak D, partial D and Del ${ }^{[6,7]}$. In general, most $\mathrm{D}$ variant alleles are assumed to be generated by gene arrangements between RHD and RHCE genes, or by single missense mutations ${ }^{[8]}$. However, it is very difficult to identify specific RHD alleles using serotyping. In this study, we used serotyping, genotyping, 


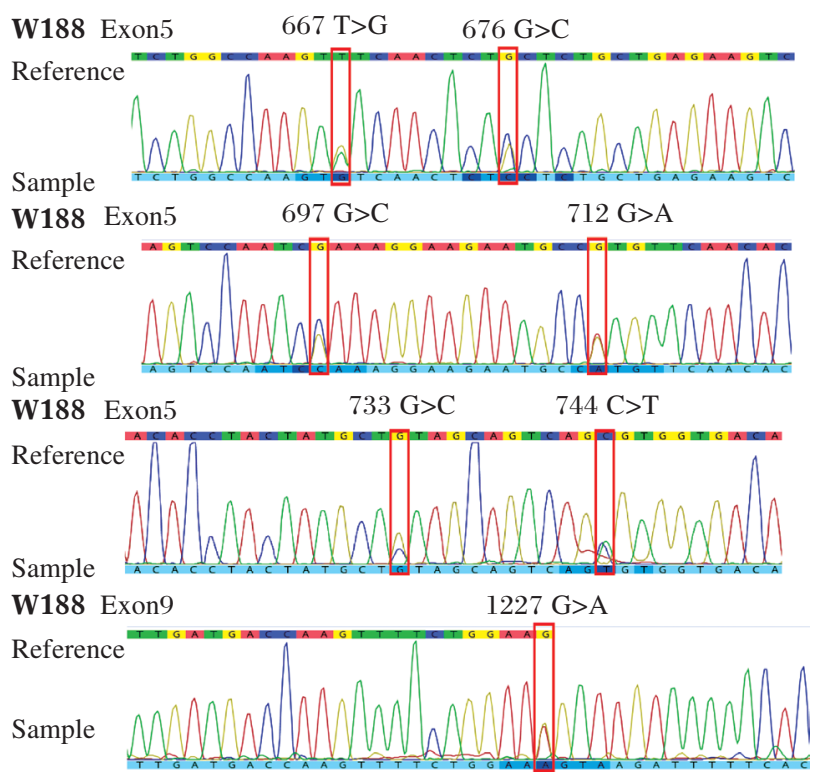

Fig. 1 RHD sequence analysis of the proband



Fig. 2 Pedigree analysis for the RHD DVa allele

sequence and pedigree analysis to confirm the very rare (RHD*05.03) allele.

RHD sequence analysis of the proband revealed the following single nucleotide polymorphisms (SNPs): $667 \mathrm{~T}>\mathrm{G}, 676 \mathrm{G}>\mathrm{C}, 697 \mathrm{G}>\mathrm{C}, 712 \mathrm{G}>\mathrm{A}, 733 \mathrm{G}>\mathrm{C}$, 744 $\mathrm{C}>\mathrm{T}$ and $1227 \mathrm{G}>\mathrm{A}$. We have no information on the proband's parents, however, the daughter's RHD sequence demonstrated $1227 \mathrm{G}>\mathrm{A}$. According to Mendel's Law of Inheritance, the novel RHD allele is $667 \mathrm{~T}>\mathrm{G}, 676 \mathrm{G}>\mathrm{C}, 697 \mathrm{G}>\mathrm{C}, 712 \mathrm{G}>\mathrm{A}$, $733 \mathrm{G}>\mathrm{C}, 744 \mathrm{C}>\mathrm{T}$. However, as yet no RHD variant allele in concordant with the novel allele exists in the Erthyrogene database ${ }^{[9]}$. Referring to RHCE and RHD reference alleles from BGMUT, $733 \mathrm{G}>\mathrm{C}$ and 744 C $>$ T are SNPs for RHD and RHCE alleles; SNPs of 733 and 744 are the specific SNPs for RHD and RHCE (733C and 744T for RHCE; 733G and 744C for RHD), which means the SNPs are not associated with the variant allele ${ }^{[10]}$. The alleles of $667 \mathrm{~T}>\mathrm{G}, 676$ $\mathrm{G}>\mathrm{C}, 697 \mathrm{G}>\mathrm{C}, 712 \mathrm{G}>\mathrm{A}$ showed to be associated with RHD*05.03 (DV type 3). The RHD allele may belong to a new allele of DV because the novel allele occurred in a variant in exon 5, while the RHD allele phenotype is consistent with DVI ${ }^{[1]}$. The results also indicated that the use of simple monoclonal anti-D to identify RHD variant is inappropriate, because of the limited data on the molecular analysis of RHD variants in the Chinese population.

RHD genotyping of the proband's RHD genetic variants were reported to be $50 \% \sim 70 \%$ RHD of the whole gene deficiency, 5.7\% RHD-RHCE(2-9)RHD hybrid and $20.2 \% 1227 \mathrm{G}>\mathrm{A}^{[7]}$. Until now, over 40 types of RHD variant have been described in Chinese individuals and alloimmunisation to anti-D is a controversial topic ${ }^{[12-18]}$. According to a report by Wang et al., in RHD25 patients who received massive transfusions of $\mathrm{RHD}^{+}$packed red blood cells, no anti-D immunisation was evident at days 168 and $232^{[19]}$. Other reports found the period of anti-D alloimmunisation to be 60 to 180 days $^{[20-24]}$. Zhou et al. described anti-D immunisation in a patient with DBT-1 allele who received $\mathrm{RHD}^{+}$packed red blood cells $^{[25]}$. Anti-D immunisation that occurs in people with RHD variants seems to be a complicated process. Due to the RHD phenotype showing negative in the $1227 \mathrm{G}>\mathrm{A}$ allele, the (RHD*05.03) phenotype is weak positive through multiple $\operatorname{IgG} / \mathrm{IgM}$ mixed monoclonal anti-D. The accurate identification of RHD variant alleles in Chinese individuals may be a necessary strategy for clinical transfusion and for pregnant women.

In conclusion, the study demonstrated a very rare RHD DVa allele and indicated that the transfusion of RHD $^{-}$blood products was a useful strategy to avoid anti-D immunisation. However, the lack of epitope identity is a strong limitation. Further pedigree analyses should be conducted.

\section{References}

[1] Daniels G. Human blood groups. London: Wiley-Blackwell, 2013:181-90.

[2] Moore BPL. Does knowledge of Du status serve a useful purpose? Vox Sang, 1984, 46(Suppl):95-7.

[3] Agre PC, Davies DM, Issit PD, et al. A proposal to standardize terminology for weak D antigen. Transfusion, 1992, 32:86-7.

[4] Gorick B, McDougall DCJ, Ouwehand WH, et al. Quantitation of D sites on selected "weak D" and "partial D" red cells. Vox Sang, 1993, 65:136-40.

[5] Wagner FF. The RHesusBase, http://www.uni-ulm. de/ wflegel/RH/.

[6] Lin YS, Chang JS, Qiu Y. Incidence of unexpected red blood cell antibodies in the north of China. Asia-Pacific Journal of Blood Types and Genes, 2017, 1(1):17-24.

[7] Yan F, Lin YS, Xu Z. Which RHD alleles are risk factors 
stimulating allo-anti-D? Asia-Pacific Journal of Blood Types and Genes, 2017, 1(3):29-32

[8] The International Society of Blood Transfusion. Red Cell Immunogenetics and Blood Group Terminology Working Party. http://www.isbtweb.org/workingparties/redcell-immunogenetics-and-blood-group-terminology.

[9] American Society of Hematology. Erythrogene: a database for in-depth analysis of the extensive variation in 36 blood group systems in the 1000 Genomes Project. http:// www.erythrogene.com/.

[10] Patnaik SK, Helmberg W, Blumenfeld OO. BGMUT: NCBI dbRBC database of allelic variations of genes encoding antigens of blood group systems. Nucleic Acids Res, 2012,40 (Database issue):D1023-9.

[11] Daniels G . Variants of RHD-current testing and clinical consequences. Br J Haematol, 2013, 161(4): 461-70.

[12] He J, YingY, HongX, et al. Molecular basis and zygosity determination of $\mathrm{D}$ variants including identification of four novel alleles in Chinese individuals. Transfusion, 2015, 55(1):137-43.

[13] Lin I, Shih M, Hsieh M, et al. Molecular basis of weak D in Taiwanese. Ann Hematol, 2003,82(10):617-20.

[14] Yan L, Wu J, Zhu F, et al. Molecular basis of D variants in Chinese persons.Transfusion, 2007,47(3):471-7.

[15] Ye L, He Y, Gao H, et al. Weak D phenotypes caused by intronic mutations in the RHD gene: four novel weak D alleles identified in the Chinese population. Transfusion, 2013,53(8): 1829-33.

[16] Ye S, Wu D, Wang M, et al. A comprehensive investigation of RHD polymorphisms in the Chinese Han popula- tion in Xi'an. Blood Transfus,2014,12(3):396-404.

[17] Chen O, Li M, Li M, et al. Molecular basis of weak D and DEL in Han population in Anhui Province, China. Chin Med J (Engl), 2012,125(18):3251-5.

[18] Ji YL, Schoot CE. Red blood cell genotyping in China. ISBT Science Series, 2016,11(Suppl.2):55-68.

[19] Wang Z, Jia S, Chen J. Epitope analysis of weak D type 25 and the transfusion strategy discussion. Chin J Blood Transfusion, 2018, 31(3): 234-7.

[20] Frohn C, Dumbgen L, Brand J, et al. Probability of anti-D development in $\mathrm{D}^{-}$patients receiving $\mathrm{D}^{+} \mathrm{RBCs}$. Transfusion, 2003, 43(7):893-8.

[21] Gunson HH, Stratton F, Phillips PK. The use of modified cells to induce an anti-RH response. Br J Haematol,1971,21(6):683-6.

[22] Pollack W, Ascari WO, Kocheskv RJ, et al. Studies on RH prophylaxis. Transfusion,1971,11(6):333-9.

[23] Samson D, Mollison PL. Effect on primary RH immunization of delayed administration of anti-RH. Immunology, 1975,28(2):349-57.

[24] Urbaniak SJ, Robertson AE. A successful program of immunizing RH-negative male volunteers for anti-D production using frozen/thawed blood. Transfusion, 1981, 21(1):64-9.

[25] Zhou X, Tian L, Song Y. DBT-1 phenotype in D variant produce anti-E, anti-D antibody and its genotype analysis: a case report. Chin J Blood Transfusion, 2018. 31(4): 428-31.

(Received 12 February 2019, Revised 21 April 2019, Accepted 08 May 2019) 